\title{
METHOD FOR UPGRADING A COMPONENT WITHIN REFURBISHMENT
}

\author{
Ganter, Nicola Viktoria; \\ Bode, Behrend; \\ Gembarski, Paul Christoph; \\ Lachmayer, Roland \\ Leibniz University Hannover
}

\begin{abstract}
One of the arguments against an increased use of repair is that, due to the constantly growing progress, an often already outdated component would be restored. However, refurbishment also allows a component to be modified in order to upgrade it to the state of the art or to adapt it to changed requirements. Many existing approaches regarding Design for Upgradeability are based on a modular product architecture. In these approaches, however, only the upgradeability of a product is considered through the exchange of components. Nevertheless, the exchange and improvement of individual component regions within a refurbishment has already been successfully carried out using additive processes. In this paper, a general method is presented to support the reengineering process, which is necessary to refurbish and upgrade a damaged component. In order to identify which areas can be replaced in the closed system of a component, the systematics of the modular product architecture are used. This allows dependencies between functions and component regions to be identified. Thus, it possible to determine which functions can be integrated into the intended component.
\end{abstract}

Keywords: Additive Manufacturing, Circular economy, Design methods, Additive Repair and Refurbishment, Modularity

\section{Contact:}

Ganter, Nicola Viktoria

Leibniz University Hannover

Institute of Product Development

Germany

ganter@ipeg.uni-hannover.de 


\section{MOTIVATION AND AIM}

For manufacturers of plant and machinery, after-sales service offers high profit margins and great potential to differentiate from competitors through reliable and fast spare parts supply (Baader et al., 2006). In order to provide spare parts, components can be newly manufactured and possibly stored for years or even decades, or components that have already been used are repaired (if necessary) and reused.

Various single case studies show that functions and characteristics of metal components can not only be restored during repair, but also improved and adapted to the state of the art. E.g., Andersson et al. (2016) show how gas turbine burners, whose tips are damaged by low cycle fatigue cracking or oxidation, can be repaired and upgraded to the latest standard. Thereby, they have been able to extend the service life of the entire burner considerably over its lifetime. The repair process involves cutting off the damaged burner tip with its delicate internal gas channels and replacing it by the latest tip design.

In this context, additive manufacturing processes offer a high degree of design freedom, which makes them particularly interesting as a technology for restoring or even improving parts with respect to existing requirements or additional functions. According to Brinker et al. (2020), the use of additive manufacturing processes to regenerate a component while retaining its function, geometry and material is referred to as Additive Repair, whereas the regeneration and simultaneous adaptation or optimization of the component as in the example above is called Additive Refurbishment. In the following, refurbishment refers to the modification or improvement of an existing part.

Current approaches, especially focussing on Additive Repair, are mainly process-based and describe the analysis of damaged parts, their pre-processing according to the chosen process, the actual additive repair process and the necessary post-processing (Jhavar et al., 2013, Sexton, 2003). Furthermore, single contributions focus on issues like bonding of melt powder to the substrate part or the design of the interface layer of such substrate parts with respect to the load conditions of the part in use (Zghair, 2019).

Focussing on Additive Refurbishment, the question arises how to support design engineers in decision-making to alter and upgrade the specification of a part and introduce new or changed functions into its geometry. Guidelines for design for upgradability mainly base on modular product structures (e.g. Ramani, 2010, Khan and Wuest, 2018). Design engineers with knowledge of the broken parts system architecture are provided with a method for its refurbishment. Based on the understanding that also single parts may be decomposed into geometric features that each fulfil functions, the authors propose to apply a modular design approach for the refurbishment of parts.

\section{RELATED WORK}

\subsection{Modularity}

A product architecture contains on the one hand the functional structure, i.e. the arrangement and the interconnections of sub-functions to form the overall function (Pahl and Beitz, 2003). On the other hand, it contains the physical product broken down into elements, which can be separable physical parts, subassemblies or distinct regions according to Ulrich (1995). The mapping between subfunctions and physical elements may be one-to-one, many-to-one or one-to-many. When a function depends strongly on two or more elements, these elements are considered strongly connected and are grouped into a module. A weak connection exists between two elements, when one has a strong influence on the fulfilment of a function while the other has a weak influence.

In addition, part of the product architecture is the specification of interfaces between interacting physical elements. Interfaces can include geometric connections as well as non-contact interactions, e.g. gear and shaft or infrared communication link. The type of interface between physical elements determines whether they are coupled or decoupled. Two components are coupled if a change in one component requires a change in the other component for the overall system to function correctly (Ulrich, 1995, Baldwin and Clark, 2000). A modular architecture comprises a one-to-one mapping and de-coupled interfaces between components. In contrast, an integral architecture includes a complex (non-one-to-one) mapping and/or coupled interfaces between components. Thus, a system, in which all elements are in a module, is a fully integrated system (Göpfert, 2009). However, the authors focus 
on system architectures for multi-component assemblies. These approaches are usually not applied on the part level.

Modular product architectures offer the advantage, among others, that processes can be parallelized, e.g. the development and testing of modules. Furthermore, a modular system can be configured by combining different variants of modules (Krause, 2018) and it is favourable to plan the variable properties within product portfolios (Gembarski and Lachmayer, 2014, Schreiber et al., 2018). Modularity or a modular architecture is thus an important prerequisite for the upgradability of a product (Xing, 2007). If upgrading shall be carried out in the context of refurbishment, existing configurations have to be reused and the improvements have to be integrated into an existing structure. Compared to the design stage, there are therefore more restrictions and fewer degrees of freedom to introduce new functions or improvements in the refurbishment. To evaluate product upgradeability in remanufacturing, Xing (2007) considers (1) the degree the existing function structure can meet and support new or changed requirements, (2) the reusability of the components for an extended service life, and (3) the structural modularity for the ease of part separation, replacement, and swap during remanufacturing processes. However, this consideration as well as the literature known to the authors is limited to the upgradability of a product through reuse or replacement of components. Upgradeability at the part level is not considered.

\subsection{Additive Refurbishment}

For decision-making regarding modification and upgrading of an existing part, a design engineer should be aware of, among other things, the potentials and degrees of freedom offered by (Additive) Refurbishment, as well as the restrictions and necessary constraints that need to be taken into account. In the following, potentials, requirements and boundary conditions of Additive Refurbishment are outlined, which were derived from application studies. As mentioned above, refurbishment offers the potential to integrate new validated design solutions from the current series into "old" components. For example, obsolete tools used for prototyping and outdated series can be upgraded to enable their reuse as production worthy tools. In addition, refurbishment allows to improve the performance of a component or tool. One example is the cycle time of injection moulding tools, which was considerably reduced in Additive Refurbishment by applying a special, thermally conductive coating system (Sexton, 2003). Furthermore, refurbishment provides the potential to increase the resistance of a component with regard to the cause of its damage. For example, the wear resistance of drilling and injection moulding tools can be increased by recreating damaged surfaces and structures using materials that have a particularly high wear resistance (Birger, 2011). In the aforementioned example of the burner tip refurbishment, Andersson et al. (2016) introduced design changes that resulted in an improvement of the temperature gradient over the tip, thus making it less exposed to low cycle fatigue cracking.

In application studies, the shape and material were exploited as degrees of freedom to achieve a desired modification or upgrade of a part. The material is usually modified with the aim of increasing the resistance to destructive chemical-corrosive or tribological effects. The degree of freedom to modify the material is strictly limited by its compatibility with the base material of the component. Moreover, only a limited number of materials is available as feedstock for the additive processes. Material combinations that have been successfully realised in Additive Repair and Refurbishment include, for instance, nickel-based alloys applied to stainless steel and the combination of different aluminium alloys (Zghair, 2017, Andersson et al., 2016).

For the decision whether and to what extent a part is refurbished, an essential question is how much of the old part is to be replaced, in other words: where to draw the line between the old and the new part. Firstly, this is determined by the damage, since all the affected material must be removed. Secondly, it must be determined how much additional material has to be removed in order to change certain existing functions or to integrate additional ones. Since a low effort for the refurbishment process (use of material etc.) is favoured, it is important to remove only as much material as necessary. Thus, design engineers are faced with the challenge of assessing the interchangeability of component areas and the expandability of a component. This requires consideration of the relationship between physical elements and the required functions, which is represented in product architectures.

Finally, defining the interface between the old and new component area requires consideration of the repair processes used. For example, for a direct material application to the part, the accessibility for the selected process must be guaranteed. With laser powder bed fusion (LPBF), e.g., a flat surface must be 
present for material application, whereas in laser deposition welding material can also be applied to free-form surfaces. In addition, attention must be paid for thin-walled structures to be damaged or distorted by heat input during the repair process. Especially in areas with filigree structures or internal cooling channels, the position of the interface must be selected according to the heat input of the selected process, since the heat input differs for different processes, e.g. tungsten inert gas welding and laser-based processes (Andersson et al., 2016).

Furthermore, when defining the interface, Zghair (2017) recommends that the operational stresses are considered in order to place the interface outside the maximum mechanical operational stresses.

Another relevant aspect for an Additive Refurbishment procedure is that the fulfillment of functions must be ensured, especially if the processes used for refurbishment were not used for the initial production of the component. For example, Andersson et al. (2016) simulatively determined that an additive refurbishment of the gas turbine burner requires the design of the flow channels to be modified. Otherwise, the required gas flow is not achieved due to the higher surface roughness of additively manufactured flow channels.

\section{METHOD}

The initial situation for the described method is a broken part and the knowledge of the parts requirements. The intension to repair also provides an option to update or upgrade the part while repairing it e.g. refurbishing.

Potentials for a refurbishment process can be identified with the modular product architecture developed design approach described e.g. by Göpfert (2009). While he described the process for assemblies, it is applied here to a single part. To do that, a part can be decomposed into elements, which are function-bound sections of a part. These elements are grouped into modules e.g. a set of strongly interacting elements. The strong connection is derived from the functions performed by the element. If two elements are needed to apply a function, these are considered strongly connected. A weak connection between two elements is present when one element contributes only marginal. In refurbishment, single modules can be replaced without a major influence on other modules. In figure 1 this is closer described by showing a generic architecture of a part. In this case, element 2 is damaged. Because element 2 is interlinked with element 1 it is part of module A. Therefore, both elements, the whole module A has to be replaced. The connection between element 2 and 3 over function 1.3 is weak. Therefore, module B does not have to be removed when replacing module A. Weak connections or no connections indicate a potential interface for the refurbishment.
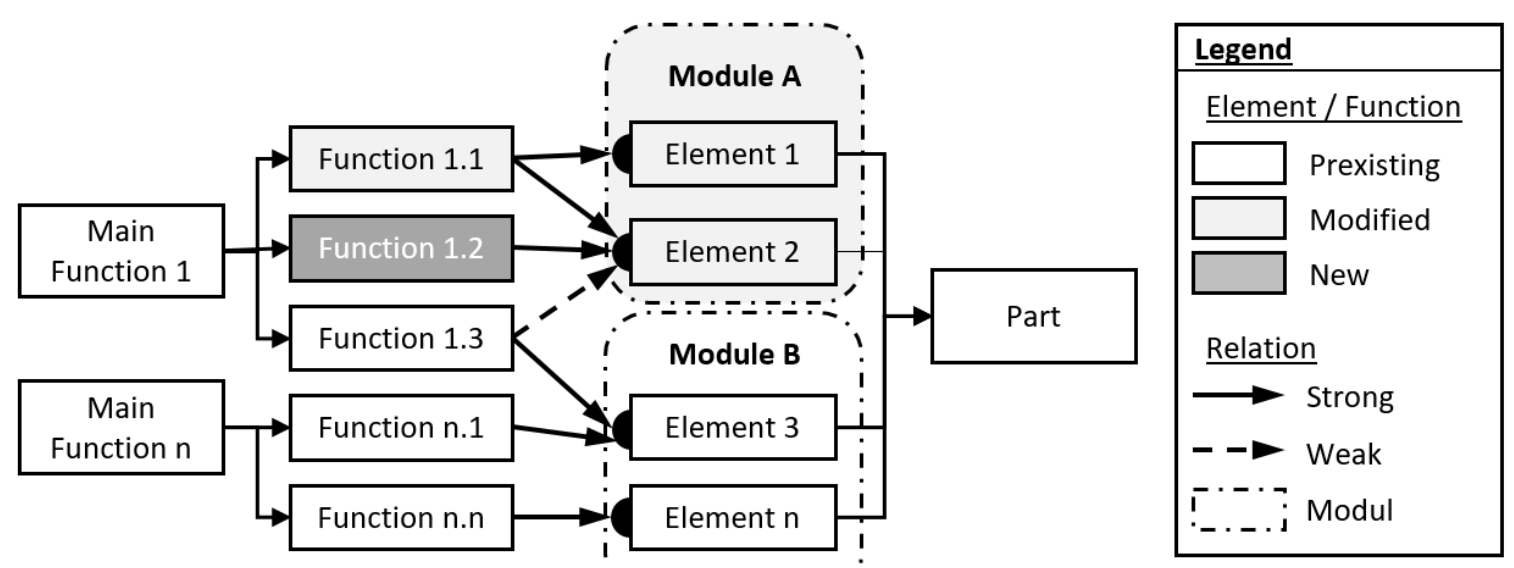

Figure 1. Product architecture, in accordance with Göpfert (2009)

Refurbishing a part essentially creates a new part. Hence, the refurbishment process can be based on the product design process. Therein, the damaged part is seen as a pre-product for the design process and manufacturing. Refurbishing a part is only considered possible if its architecture can be derived. This implies that the functions the part is able to perform are well known. Additionally, the part needs at least one "good" module that is worth keeping. The damaged part must therefore consist out of more than one module (not fully integrated). Furthermore, the material of the part must be known or identified in order to choose a suitable repair process chain accordingly. In addition, the geometry of 
the damaged part based on CAD models or technical drawings must be known or captured, e.g. by $3 \mathrm{~d}$ scanning.

Looking at the function side of the product architecture, refurbishment offers four possibilities for each function:

1. Keeping respectively restoring

2. Removing

3. Improving

4. Adding new functions

In refurbishment and repair at least one function must be kept. Removing a function can be done for replacing an existing function with another one or moving the function into another part. Option 3 and 4 can be enabled by replacing a module. This is helpful if there is a newer product architecture to update to. This demands both architectures to have corresponding modules.

To avoid the terms "old" and "new" when talking about product iterations they will be called architecture 1.0 and architecture 2.0. The refurbishment process is presented in figure 2. Starting with the damaged part (1), a failure analysis (4) needs to be performed. Here, the damaged and undamaged regions as well as the cause of failure need to be identified. This can range from a simple experiencebased identification to an extensive analysis of material, geometry and/or usage. If no system architecture 2.0 is available (5) or architecture 2.0 is available but the cause of failure is not solved (6), knowledge of the failure can be used (8) to update the architecture (9) and avoid this particular failure case in future products (9). A valuable tool for the update of the new architecture are design catalogues to develop solutions. Alternatively, if the architecture 2.0 (3) already solved the issue (6), these steps can be skipped. After the failure analysis the failure is being matched (7) to an element based on the architecture 1.0 (2). In the next step, the two architectures 1.0 (2) and 2.0 (3) respectively 1.1/2.1 (9) have to be compared. Functions are being picked from to the newest architecture and prioritized (10) to circumvent possible failures, comply to new regulations or to meet a certain goal. Depending on the goals and complexity different methods like $\mathrm{ABC}$ analysis, benefit analysis, pair-by-pair comparison or SWOT analysis can be used to objectify the decision. Regarding the product architecture it should be considered, which element performs which functions. Changing a function implies changing the whole module. Based on the picked functions and the component characteristics, such as dimensions or material, an appropriate repair process needs to be chosen (11). This also has an impact on the modules that have to be removed. Depending on the selected repair method, modules may have to be removed due to their location in the component, e.g. if LPBF is applied, because they are on the same plane as damaged modules. This finally decides which functions are being implemented in the refurbished design. In order to select a suitable manufacturing process for refurbishment, the characteristics of the component must be compared with the restrictions of the various processes, e.g. whether the size of the component allows it to be processed within the building space of an LPBF system. In addition, the effective principles intended by the functional upgrade must be realisable with the selected process. Another criterion is a reduction of the refurbishment effort (e.g. little material has to be removed in order to create accessibility for the additive material application or post-processing steps have to be avoided). The knowledge required to select suitable processes for refurbishment is available, for example, from system data sheets or scientific literature. For example, Kranz (2017) and Lippard (2018) list limit values for structures that can be produced in LPBF. If no information can be obtained from the literature, e.g. regarding the compatibility of two materials, proof of concept tests are carried out before a process is finally selected.

After this step, a new architecture "version 3.0" (12) is defined (13). Only the new modules for which no preliminary drafts exist are then designed. From here, we can jump into the general design process, e.g. VDI 2221 (2019) (14), where most of the modules are already designed. In the next steps, the remaining modules are designed, combined to the overall part and documented. The modular approach has the advantage that many modules are already validated. Also new modules can be developed and validated (simulatively and/or experimentally) in parallel due to their relative independency. This saves time and resources. A holistic validation is still necessary. 


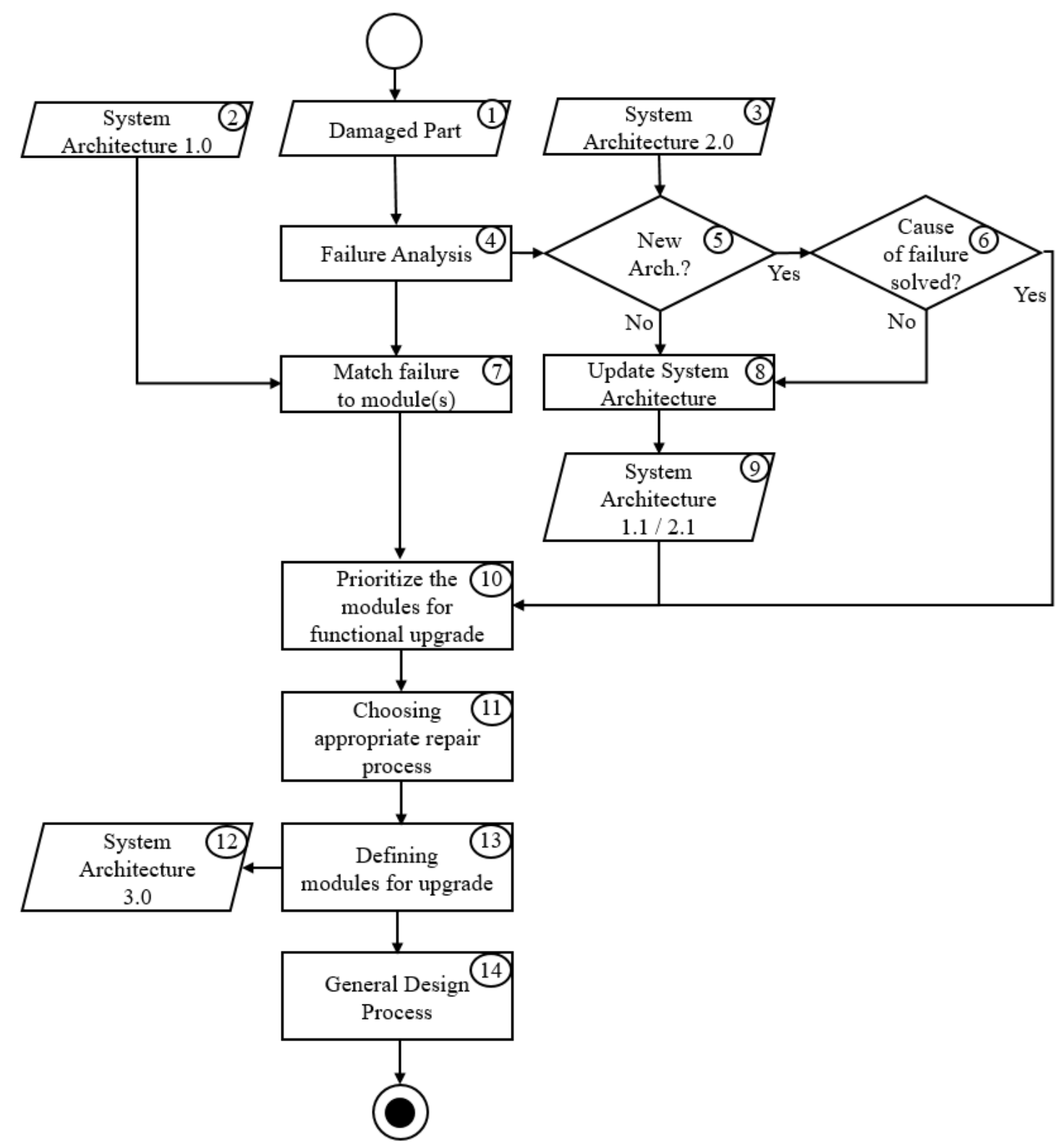

Figure 2. Method for refurbishment 


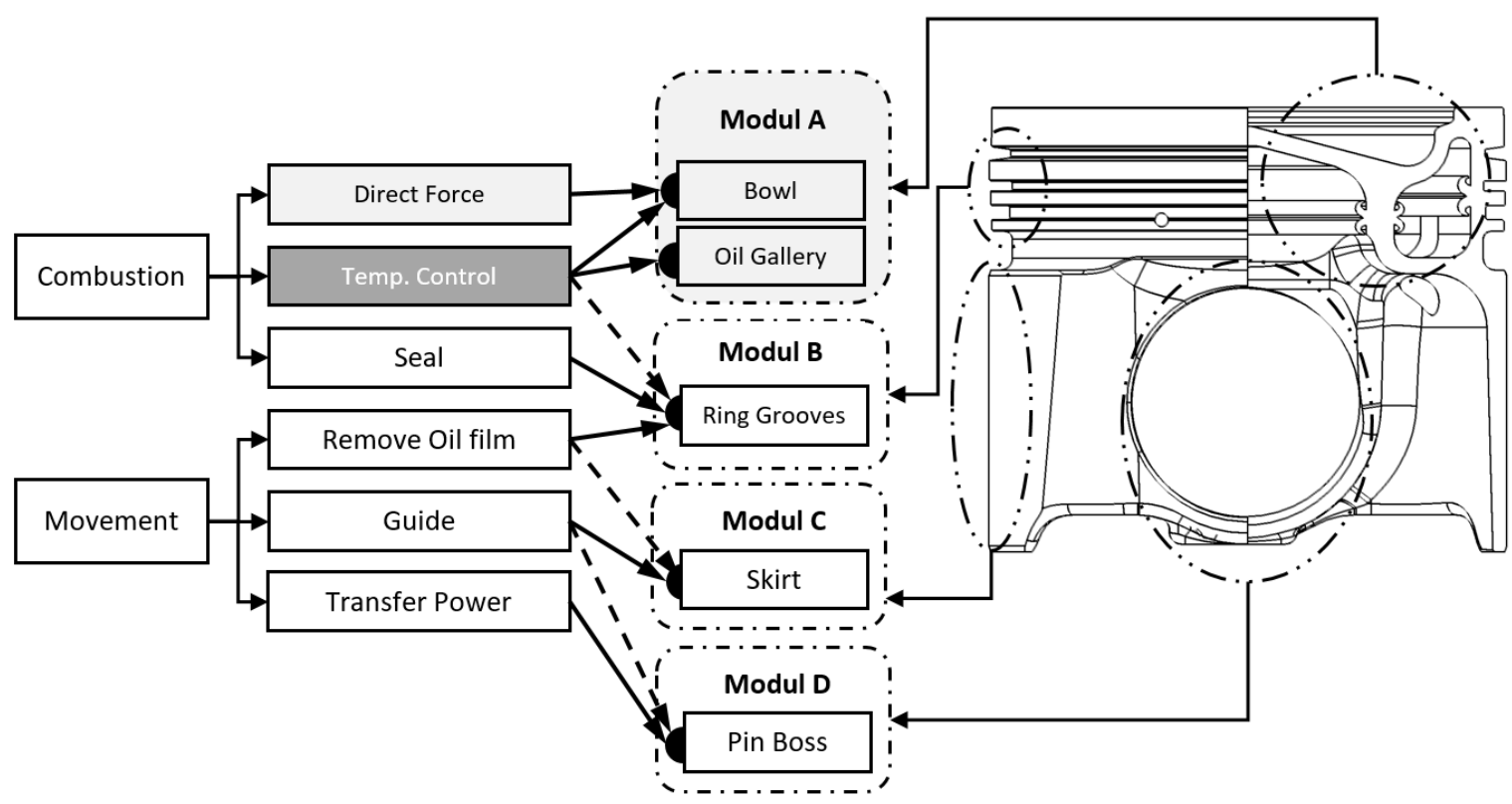

Figure 3. System architecture piston

In this hypothetical example, a piston of an internal combustion engine is on the edge of failure due to a crack in the piston head. The developer of this piston, knowing the system architecture (shown in figure 3 above) as well as the CAD model and the material, can decide to refurbish his broken pistons. A piston is usually defined by thousands of requirements, these are not in focus of this paper so two main functions have been derived to explain the procedure. These are the enduring and directing of the combustion caused by the injection of fuel and the oscillating movement. The derived sub functions are then matched to elements. The cross section of the described piston is on the right side and is dissected into its elements. Notably the temperature control of the piston is mainly done by the piston bowl absorbing the heat from the combustion and the oil filled cooling gallery absorbing approx. 75\% of the combustion heat, while the rest going through the ring grooves, skirt and pin (Pan, 2005). Therefore, the connections between the bowl and the cooling gallery through the sub function temperature control is strong, whereas the connection between these two and the ring grooves is weak. Bowl and cooling gallery are combined into module A. Each other element is in its own module due to no strong connections through a sub function. Although being a solid, one-piece piston this part can be seen as a modular system with five elements being in four modules. The results of the failure analysis are that the cooling gallery was not suitable for the bowls high temperatures. The function "Temperature Control" was therefore the cause of the failure. The other elements in this scenario are fine. For this reason, module $\mathrm{A}$ is to be replaced within a refurbishment in order to realise a temperature control that is suitable for the temperatures of the bowl. For the arguments sake the failure is already addressed in the existing system architecture 2.0. The new architecture includes an optimized piston bowl and cooling gallery, the ring grooves and skirt remain the same. The pin bore in architecture 2.0 got smaller. To meet the current emission standards the new bowl and cooling gallery need to be implemented in refurbishment. This also fixes the cause for the piston bowl crack. The smaller pin bore reduces friction but due to the robust bearing in architecture 1.0 the function is not of such a high priority. The filigree structures required in the cooling channels are a decisive criterion for the choice of the process. Since LPBF enables the filigree structures to be created and other component characteristics such as the material or the component size likewise allow processing, LPBF is selected. For the final determination of the modules to be removed, consideration is given to the fact that the ring grooves are in the same plane as module A. Since a flat plane is required for applying material by LPBF, not only the damaged module but also the ring grooves have to be removed by $\mathrm{CNC}$ milling before the LPBF process. All functions regarding module $\mathrm{A}$ and $\mathrm{B}$ are taken from architecture 2.0 for architecture 3.0. A general design process can be started with the modules $\mathrm{C}$ and $\mathrm{D}$ already designed wherein, among other things, the interfaces between modules are designed. Special care must be given to the connection between the initial part and the printed attachment. Here, 
verification by means of experiments and simulation is necessary. In addition, functional requirements such as fatigue strength need to be verified by simulation and/or experiment due to the changes in part properties caused by the repair process, such as microstructure or surface roughness.

\section{DISCUSSION}

The developed method represents a procedure to support the reengineering process, which is necessary to refurbish a damaged component.

On the application example of a piston, which has a multitude of constraints and connections between physical modules and functions, the method is applied successfully. However, for the applicability of the method to the piston, the damage case is very decisive. In the considered case, only one module is damaged by the present hot crack. But if a crack is more advanced, so that all modules of the piston have to be replaced, refurbishing is not practical. This is also the case if the component has a large number of severe damages affecting all modules. Furthermore, the application of the method is complicated or even impossible for a highly integral component or if the system architecture of the new component generation is highly integral.

Considerable know-how is required to determine whether links between functions and physical elements are weak and thus negligible, allowing these elements to be changed in the refurbishment process. In this context, assumptions made regarding the links between physical elements and functions should be supported and validated by analysis.

The perspective taken in this paper that a damaged component provides modules for further use can be applied to the development process. In other words, a damaged component can be seen as a "semifinished product" for the production of a new component. In order to refurbish components, it is sensible to determine during product development phase whether it is a suitable maintenance strategy, e.g. additive refurbishment using LPBF. In this case, module interfaces are defined in the component that remain consistent for different component variants and across generations. These interfaces can reduce the effort for the intended refurbishment process and thus increase the upgradability of the component.

The use of additive refurbishment as a maintenance strategy can be suitable for components wherein one area is highly stressed and therefore fails much earlier than other areas, as in the example of the burner tip. In addition, refurbishment can be provided during product development when it is known that the state of the art or the requirements for a particular component area change between product generations.

By introducing a new state of the art or changed requirements into existing components, it enables a continuous improvement of existing machines and plants. Furthermore, it allows obsolete components to be used for new product generations.

\section{CONCLUSION AND OUTLOOK}

A method to assist the refurbishment of a part has been presented. Based on an outdated product architecture and a broken part a failure analysis is proposed that allows to circumvent this cause for a potential breakdown. From this basis, new and modified functions can be derived and integrated in an updated system architecture for the refurbished part.

Based on the designer's demands and manufacturing restrictions, functions and corresponding modules can be included. While previous approaches were only applied at the assembly level, this approach allows a systematic process to perform an upgrade on component level. Thus, this approach provides methodological support for the application of Additive Refurbishment in the future. To further validate the method, it is necessary to realize the refurbishment process of the presented application example and test whether the created part fulfils all requirements. Further work should investigate when Additive Refurbishment is economically and ecologically beneficial. For this purpose, the product life cycle costs and life cycle assessment of the original component must be compared with the refurbished component in various case studies. Furthermore, design guidelines should be developed with the aim of ensuring a "design for refurbishment" in future products. 


\section{ACKNOWLEDGMENTS}

This research was conducted in the scope of the research project RePARE- Regeneration of product and production systems through additive repair and refurbishment (Funding reference number 033R229) which is funded by Federal Ministry of Education and Research (BMBF) within the funding measure "Resourceefficient Circular Economy - Innovative Product Cycles" (ReziProK).

\section{REFERENCES}

Andersson, O., Graichen, A., Brodin, H. and Navrotsky, V. (2016), "Developing Additive Manufacturing Technology for Burner Repair”, in Proceedings of ASME Turbo Expo 2016: Turbomachinery Technical Conference and Exposition, June 13 - 17, 2016, Seoul, South Korea. https://doi.org/10.1115/gt2016-56594

Baader, A., Montanus, S., Sfat, R. (2006) "After Sales Services — mit produktbegleitenden Dienstleistungen profitabel wachsen”, In: Barkawi, K., Baader, A., Montanus, S. (Eds.) Erfolgreich mit After Sales Services. Springer, Berlin, Heidelberg. https://doi.org/10.1007/3-540-34548-5_1

Baldwin, C. Y. \& Clark, K. B. (2000). "Design rules: The power of modularity" (Vol. 1). MIT press. https://doi.org/10.7551/mitpress/2366.001.0001

Birger, E. M., Moskvitin, G.V., Polyakov, A.N. and Arkhipov, V.E. (2011), "Industrial laser cladding: current state and future", in Welding International, Vol. 25, pp. 234-243. https://doi.org/10.1080/09507116.2010.540880

Brinker, J., Gembarski, P.C., Hagen, S. and Thomas, O. (2020), “Anwendungspotenziale von Additive Repair und Refurbishment für Service-orientierte Geschäftsmodelle”, in Lachmayer, R., Rettschlag, K., Kaierle, S. (Eds.) Konstruktion für die Additive Fertigung 2019, pp. 43-54. https://doi.org/10.1007/978-3-662-61149-4_4

Gembarski, P. C., Lachmayer, R. (2014), "Forward variance planning and modeling of multi-variant products", Procedia CIRP, 21, 81-86. https://doi.org/10.1016/j.procir.2014.03.161

Göpfert, J. (2009). "Modulare Produktentwicklung-Zur gemeinsamen Gestaltung von Technik und Organisation; Theorie- Methodik- Praxis”, Zugl.: München, Univ., Diss., 1998, ID-Consult Wissen für die Praxis, 2. Aufl., Books on Demand, Norderstedt. https://doi.org/10.1007/978-3-663-08152-4_3

Jhavar, S., Paul, C.,P., Jain, N. K. (2013), "Causes of failure and repairing options for dies and molds: A review”, Engineering Failure Analysis, Vol.34, pp.519-535. https://doi.org/10.1016/j.engfailanal.2013.09.006

Khan, M.A., Wuest, T. (2018), “Towards a framework to design upgradable product service systems", Procedia CIRP, Vol. 78, pp. 400-405. https://doi.org/10.1016/j.procir.2018.08.326

Krause, D., Gebhardt, N. (2018), "Methodische Entwicklung modularer Produktfamilien: hohe Produktvielfalt beherrschbar entwickeln”, Springer-Verlag. https://doi.org/10.1007/978-3-662-53040-5

Pahl, G., Beitz, W., Schulz, H. J., Jarecki, U. (2013), "Pahl/Beitz Konstruktionslehre: Grundlagen erfolgreicher Produktentwicklung. Methoden und Anwendung.”, Springer-Verlag. https://doi.org/10.1007/978-3-66257303-7

Pan, J., Nigro, R., Matsuo, E. (2005), “3-D modeling of heat transfer in diesel engine piston cooling galleries", SAE transactions, 1174-1181. https://doi.org/10.4271/2005-01-1644

Ramani, K., Ramanujan, D., Bernstein, W. Z., Zhao, F., Sutherland, J., Handwerker, C., Choi, J., Kim, H., Thurston, D. (2010), “Integrated sustainable life cycle design: a review.” Journal of Mechanical Design 132.9. https://doi.org/10.1115/1.4002308

Schreiber, D., Gembarski, P.C., Lachmayer, R. (2018), "Data Models for PSS Development and Configuration: Existing Approaches and Future Research”, In: Hankammer, S., Nielsen, K., Piller, F. Th., Schuh, G., Wang, N. (Eds.) Customization 4.0, Springer International Publishing, pp. 55-74. https://doi.org/10.1007/978-3-319-77556-2_4

Sexton, L. (2003), "Laser cladding: repairing and manufacturing metal parts and tools", in Proc. SPIE 4876, Opto-Ireland 2002: Optics and Photonics Technologies and Applications. https://doi.org/10.1117/12.463704

Ulrich, K. (1995). "The role of product architecture in the manufacturing firm”, Research policy, 24(3), 419-440. https://doi.org/10.1016/0048-7333(94)00775-3

Xing, K., Belusko, M., Luong, L. and Abhary, K. (2007), “An evaluation model of product upgradeability for remanufacture", The International Journal of Advanced Manufacturing Technology, Vol. 35 No. 1-2, pp. 114. https://doi.org/10.1007/s00170-006-0698-9

Zghair, Y.A., Leuteritz, G. (2017), “Additive Repair von Multimaterialsystemen im Selektiven Laserstrahlschmelzen”. In: Lachmayer R., Lippert R. (Eds.) Additive Manufacturing Quantifiziert. Springer Vieweg, Berlin, Heidelberg. https://doi.org/10.1007/978-3-662-54113-5_13

Zghair, Y.A. (2019), “Additive Repair Design Process for Aluminium Components”, Dissertation, Institut für Produktentwicklung (IPeG), Leibniz Universität Hannover, Hannover, 2019. 


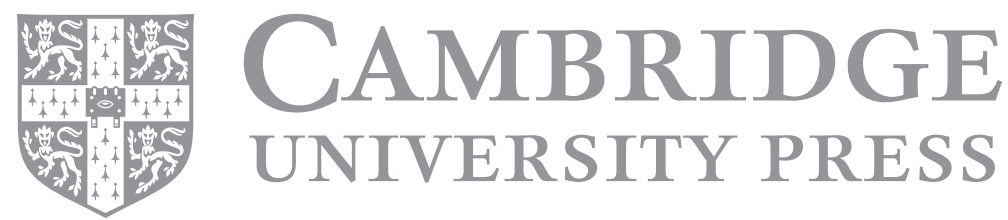

